Article

\title{
Lymphopenia as a Predictor for Adverse Clinical Outcomes in Hospitalized Patients with COVID-19: A Single Center Retrospective Study of 4485 Cases
}

\author{
Jianli Niu $^{1}{ }^{(D)}$, Candice Sareli ${ }^{1}$ (D), Daniel Mayer ${ }^{2}$, Alvaro Visbal $^{2}$ and Aharon Sareli ${ }^{2, *}$ \\ 1 Office of Human Research, Memorial Healthcare System, Hollywood, FL 33021, USA; JNiu@mhs.net (J.N.); \\ CSareli@mhs.net (C.S.) \\ 2 Adult Critical Care Service, Memorial Healthcare System, Hollywood, FL 33021, USA; \\ DMayer@mhs.net (D.M.); AVisbal@mhs.net (A.V.) \\ * Correspondence: ASareli@mhs.net; Tel.: +1-954-265-9976
}

Citation: Niu, J.; Sareli, C.; Mayer, D.; Visbal, A.; Sareli, A. Lymphopenia as a Predictor for Adverse Clinical Outcomes in Hospitalized Patients with COVID-19: A Single Center Retrospective Study of 4485 Cases. J. Clin. Med. 2022, 11, 700. https:// doi.org/10.3390/jcm11030700

Academic Editor: Shinichi Sato

Received: 6 January 2022

Accepted: 27 January 2022

Published: 28 January 2022

Publisher's Note: MDPI stays neutral with regard to jurisdictional claims in published maps and institutional affiliations.

Copyright: (C) 2022 by the authors. Licensee MDPI, Basel, Switzerland. This article is an open access article distributed under the terms and conditions of the Creative Commons Attribution (CC BY) license (https:// creativecommons.org/licenses/by/ $4.0 /)$.

\begin{abstract}
Lymphopenia is commonly present in patients with COVID-19. We sought to determine if lymphopenia on admission predicts COVID-19 clinical outcomes. A retrospective chart review was performed on 4485 patients with laboratory-confirmed COVID-19, who were admitted to the hospital. Of those, 2409 (57.3\%) patients presented with lymphopenia (absolute lymphocyte count $\left.<1.1 \times 10^{9} / \mathrm{L}\right)$ on admission, and had higher incidences of ICU admission $(17.9 \%$ versus $9.5 \%$, $p<0.001)$, invasive mechanical ventilation $(14.4 \%$ versus $6.5 \%, p<0.001)$, dialysis $(3.4 \%$ versus $1.8 \%, p<0.001)$ and in-hospital mortality $(16.6 \%$ versus $6.6 \%, p<0.001)$, with multivariable-adjusted odds ratios of 1.86 (95\% confidence interval [CI], 1.55-2.25), 2.09 (95\% CI, 1.69-2.59), 1.77 (95\% CI, 1.19-2.68), and 2.19 (95\% CI 1.76-2.72) for the corresponding outcomes, respectively, compared to those without lymphopenia. The restricted cubic spline models showed a non-linear relationship between lymphocyte count and adverse outcomes, with an increase in the risk of adverse outcomes for lower lymphocyte counts in patients with lymphopenia. The predictive powers of lymphopenia, expressed as areas under the receiver operating characteristic curves, were $0.68,0.69,0.78$, and 0.79 for the corresponding adverse outcomes, respectively, after incorporating age, gender, race, and comorbidities. In conclusion, lymphopenia is a useful metric in prognosticating outcomes in hospitalized COVID-19 patients.
\end{abstract}

Keywords: lymphocyte count; lymphopenia; adverse clinical outcomes; COVID-19; restricted cubic splines; receiver operating characteristic curves

\section{Introduction}

The COVID-19 pandemic, caused by the severe acute respiratory syndrome coronavirus 2 (SARS-CoV-2), remains a global public healthcare challenge. The clinical course of COVID-19 ranges from asymptomatic disease to critical illness and death [1-4]. The risk of poor outcomes associated with COVID-19 appears to increase with old age, male gender, smoking status and the coexistence of comorbidities such as obesity, hypertension, diabetes, cardiovascular disease, chronic kidney disease, chronic obstructive pulmonary disease, and malignancy [3-7]. Several inflammatory response markers including procalcitonin, serum ferritin, C-reactive protein (CRP), D-dimer, and interleukin-6 (IL-6), which have been found to correlate significantly with severity of disease and poorer outcomes of COVID-19 patients $[8,9]$. However, the use of common clinical characteristics and serum biomarkers has significant limitations in reliably predicting clinical outcomes such as admission intensive care unit (ICU), need for mechanical ventilation, and death in hospitalized COVID-19 patients $[10,11]$. Given the recurrence of high prevalence SARS-CoV-2 waves in the United States, prognostication and risk assessments of adverse outcomes in COVID-19 patients are key priorities. 
Lymphocytes play a pivotal role in the immune response against viral infections [12]. A decrease in lymphocyte counts has been described in many respiratory infections caused by viruses [13,14]. Importantly, a low lymphocyte count has been proposed as a worse prognostic marker in patients with influenza virus infection $[15,16]$, chronic obstructive pulmonary disease [17], heart diseases [18,19], and malignancy [20]. In patients infected with SARS-CoV-2, a low lymphocyte count in peripheral blood is a common clinical feature, particularly in patients with severe COVID-19 [2,21,22]. Thus far, several studies have suggested that the degree of lymphocyte count reduction correlates with illness severity in patients with COVID-19 $[8,22,23]$. It remains uncertain whether a low lymphocyte count at admission in COVID-19 patients may signify a risk of subsequent adverse clinical consequences. Furthermore, the extent to which lymphopenia is associated with poor clinical outcomes merits further definition. Herein we performed a retrospective observational study of a large cohort of adult COVID-19 patients hospitalized at the Memorial Healthcare System, Hollywood, Florida, between 7 March 2020 and 18 January 2021, to test the hypothesis that lymphopenia has the prognostic capacity for adverse clinical outcomes in hospitalized COVID-19 patients. We investigated the relationship between the continuous changes of absolute lymphocyte count (ALC) on admission and the risk of developing various adverse clinical outcomes such as requirement for admission to the ICU, use of invasive mechanical ventilation, requirement of dialysis due to acute kidney injury (AKI), and in-hospital mortality in COVID-19 patients. We also explored the correlations between the lymphopenia and serum inflammatory biomarkers in hospitalized COVID-19 patients. In addition, we tested whether the prognostic capacity of lymphopenia is strengthened by the incorporation of other known risk factors associated with poor outcomes in COVID-19 patients.

\section{Materials and Methods}

\subsection{Study Population and Design}

This was a single center, retrospective, observational study of consecutive patients who were hospitalized with a diagnosis of COVID-19 at any of the four adult hospitals of the Memorial Healthcare System (MHS) in Hollywood, Florida from 7 March 2020 to 18 January 2021. COVID-19 diagnosis in laboratory-confirmed patients were based on polymerase chain reaction (PCR) tests. Patients with positive SARS-CoV-2 PCR tests were included in the study. Patients were excluded from the study if they were (1) age under 18 years; (2) missing absolute lymphocyte count; (3) hospitalization was less than 24 h; (4) known HIV and/or hematologic disease; and (4) ALC $>5.0 \times 10^{9} / \mathrm{L}$. This study was approved by the Institutional Review Board of MHS (protocol No. MHS.2020.065). Written informed consent was waived as this was a retrospective analysis of existing data.

\subsection{Data Collection}

Data were obtained from patients' electronic medical records. Demographic characteristics included age, gender, race/ethnicity, and underlying conditions such as obesity (defined as a calculated body mass index $\geq 30 \mathrm{~kg} / \mathrm{m}^{2}$ ), smoking status, hypertension, diabetes, chronic obstructive pulmonary disease (COPD), coronary heart disease, chronic kidney disease, and malignancy. Laboratory results, including ALC and serum levels for CRP, D-dimers, IL-6, lactate dehydrogenase (LDH), and creatinine obtained within $24 \mathrm{~h}$ of hospitalization were used in the study.

All patients in the study were followed from admission to death or to discharge from the hospital. For patients with multiple admissions during the study period, the most severe outcome was assigned. The primary outcome of this study was all-cause mortality during hospitalization. Secondary outcomes included requirement for admission to the ICU, use of invasive mechanical ventilation, and requirement of dialysis due to AKI. Lymphopenia was defined as an ALC $<1.1 \times 10^{9} / \mathrm{L}\left(1100\right.$ cells $\left./ \mathrm{mm}^{3}\right)$ based on a previous meta-analysis showing a consistent outcome in four studies using the same cutoff point [21]. 


\subsection{Statistical Analysis}

Descriptive statistics were used to summarize characteristics and outcomes from the study population. Continuous variables were presented using median and interquartile range (IQR) or mean \pm standard deviation (SD), whereas categorical variables were presented using absolute number and frequencies (\%). Comparisons between groups were performed using independent sample $t$-tests, Mann-Whitney U-tests, Chi-square or Fischer's exact tests, as appropriate. The Spearman rank test was used to analyze the correlations between ALC and serum levels of CRP, IL-6, LDH, D-dimer, and creatinine. The Kendall's tau-b test was performed to measure the correlations between ALC and the adverse outcomes of interest, including in-hospital mortality, requirement for ICU admission, use of invasive mechanical ventilation, and requirement for dialysis.

Multivariable logistic regression models were constructed to estimate the odds ratios (ORs), adjusted ORs and their $95 \%$ confidence intervals (CIs) for association of lymphopenia with the four outcomes of interest. Covariates for the various models were chosen based on a prior review of the literature and clinical judgment, focusing on variables that might be expected to confound the lymphopenia-risk relationship. Models were initially adjusted for age and gender (model A), and subsequently additionally adjusted for the known risk factors including race/ethnicity, hypertension, diabetes, COPD, chronic kidney disease, coronary artery disease, malignancy, obesity, and smoking (model B). Restricted cubic splines (RCS) [24] with four knots at the 25th, 50th, 75th, and 95th percentiles of ALC distribution were incorporated into the logistic models to flexibly estimate risks for the outcomes of interest based on the continuous changes of lymphocyte counts. The results were visually presented with graphs by entering the lymphocyte count as a continuous variable into the logistic regression analysis, with a reference point at $1.1 \times 10^{9} / \mathrm{L}$. These analyses were adjusted for the variables in model B. The receiver operating characteristic (ROC) curves were then determined to determine the prognostic power of lymphopenia alone or adjusted with other clinical variables for predicting the outcomes of interest. Differences in ROC area under curve (AUC) were tested using the method of Delong et al. [25]. Statistical analyses were performed using the Statistical Package for the Social Sciences (SPSS version 27; Chicago, IL, USA), STATA version 15 (StataCorp, LLC, College Station, TX, USA). A $p$ value of less than 0.05 was considered statistically significant.

\section{Results}

Between 7 March 2020 and 18 January 2021, a total of 4870 adult patients were admitted to the MHS for COVID-19. After excluding five patients who died during the first $24 \mathrm{~h}$ after admission, 47 patients who had HIV, 325 patients with unavailable lymphocyte counts in the electronic medical record, and eight patients with lymphocyte counts $>5.0 \times 10^{9} / \mathrm{L}$ (considering that they could suffer from a concomitant lymphoproliferative disorder), we included 4485 patients with COVID-19 in the final analysis (Figure 1). 


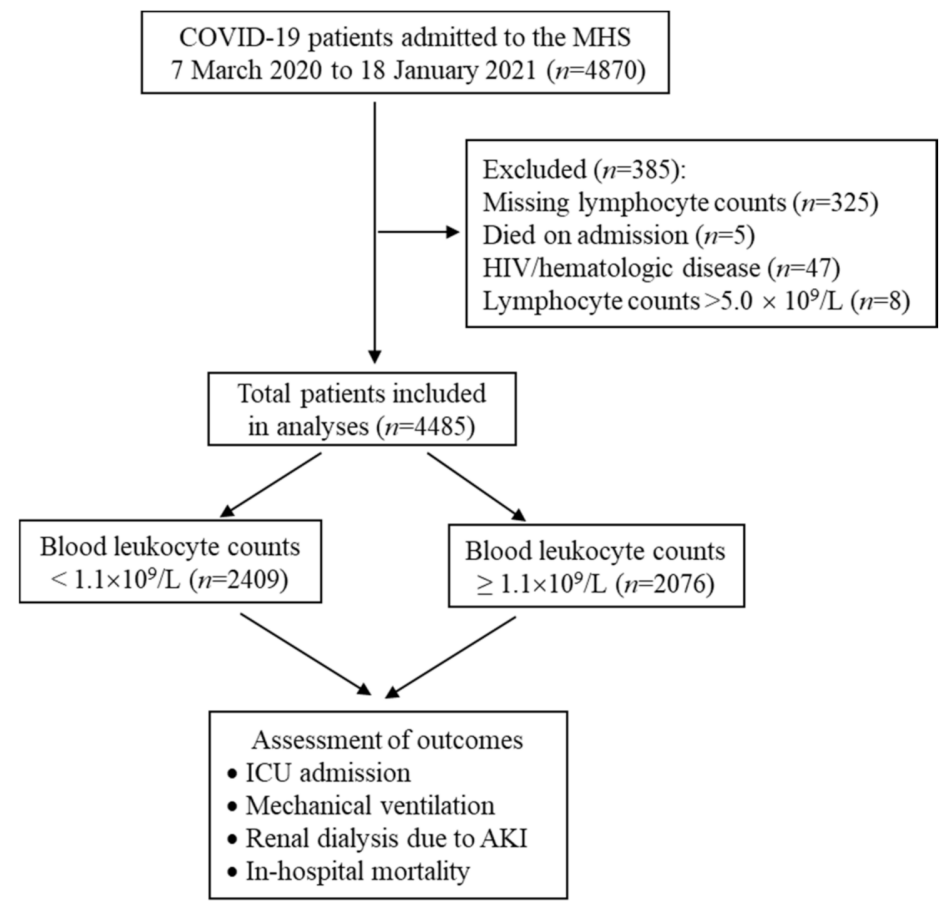

Figure 1. Flow chart of study population and clinical course assessment. Lymphocytes were determined using enrollment complete blood counts. ICU, intensive care unit; AKI, acute kidney failure.

\subsection{Patient Characteristics Associated with Lymphopenia}

At the time of hospital admission, the mean absolute lymphocyte count of the study population was $1.18(\mathrm{SD}, 0.62) \times 10^{9} / \mathrm{L}$ with a median of $1.1(\mathrm{IQR}, 0.7-1.5) \times 10^{9} / \mathrm{L}$. Table 1 summarizes the baseline characteristics of 4485 patients stratified by lymphopenia (defined as an ALC $<1.1 \times 10^{9} / \mathrm{L}$ ). A total of 2409 (53.7\%) patients had lymphopenia, and $2076(46.3 \%)$ patients had no lymphopenia. Compared with patients without lymphopenia, patients with lymphopenia tended to be significantly older, more male and Hispanic. This subset of patients also had a significantly higher comorbidity profile including hypertension, chronic obstructive pulmonary disease, chronic kidney disease, coronary artery disease, obesity, and smoking. Diabetes mellitus and malignancy were two comorbidities that did not significantly differ between patients with lymphopenia and without. Laboratory testing on admission revealed that patients with lymphopenia had higher levels of CRP, IL-6, LDH, and creatinine compared to patients without lymphopenia.

Table 1. Baseline characteristics of patients stratified by absolute lymphocyte count.

\begin{tabular}{lcccc}
\hline \multirow{2}{*}{ Variables } & Total Patients & \multicolumn{2}{c}{ Absolute Lymphocyte Count } & \multirow{2}{*}{$p$ Value } \\
\cline { 3 - 4 } & & $<\mathbf{1 . 1} \times \mathbf{1 0}$ / $/ \mathbf{L}$ & $\mathbf{1 . 1} \times \mathbf{1 0} / \mathbf{L}$ & \\
\hline Patients, n (\%) & 4485 & $2409(53.7)$ & $2076(46.3)$ & \\
Age, years & $60(46-72)$ & $64(52-75)$ & $57(41-70)$ & $<0.001$ \\
Male, n (\%) & $2311(51.5)$ & $1413(58.7)$ & $898(43.3)$ & $<0.001$ \\
Race, n (\%) & & & & $<0.001$ \\
White & $756(16.9)$ & $451(18.7)$ & $305(14.8)$ & \\
Black & $1358(30.3)$ & $593(24.6)$ & $765(36.8)$ & \\
Hispanic & $2146(47.8)$ & $1244(51.6)$ & $902(43.4)$ & \\
Asian & $49(1.1)$ & $31(1.3)$ & $18(0.9)$ & \\
Other & $176(3.9)$ & $90(3.7)$ & $86(4.1)$ & \\
\hline
\end{tabular}


Table 1. Cont.

\begin{tabular}{|c|c|c|c|c|}
\hline \multirow{2}{*}{ Variables } & \multirow{2}{*}{ Total Patients } & \multicolumn{2}{|c|}{ Absolute Lymphocyte Count } & \multirow{2}{*}{$p$ Value } \\
\hline & & $<1.1 \times 10^{9} / \mathrm{L}$ & $\geq 1.1 \times 10^{9} / \mathrm{L}$ & \\
\hline \multicolumn{5}{|l|}{ Comorbidity, n (\%) } \\
\hline Diabetes & 1813 (40.4) & 1000 (41.5) & $813(39.2)$ & 0.109 \\
\hline Hypertension & $2953(65.8)$ & $1701(70.6)$ & $1252(60.3)$ & $<0.001$ \\
\hline COPD & $374(8.3)$ & $234(9.7)$ & $140(6.7)$ & $<0.001$ \\
\hline Chronic kidney disease & $670(14.9)$ & $399(16.6)$ & $271(13.1)$ & $<0.001$ \\
\hline Coronary heart disease & 705 (15.7) & $420(17.4)$ & $285(13.7)$ & $<0.001$ \\
\hline Malignancy & $231(5.2)$ & $136(5.7)$ & $95(4.6)$ & 0.106 \\
\hline Obesity & $1061(23.7)$ & 835 (34.7) & $226(10.9)$ & $<0.001$ \\
\hline Smoking & $1089(24.3)$ & $623(25.9)$ & $466(22.4)$ & 0.008 \\
\hline \multicolumn{5}{|l|}{ Laboratory testing } \\
\hline $\mathrm{CRP}, \mathrm{mg} / \mathrm{L}$ & $6.6(2.8-12.1)$ & $7.4(3.4-12.9)$ & $5.6(2.2-10.9)$ & $<0.001$ \\
\hline D-dimer, mg/L & $0.9(0.5-1.8)$ & $0.9(0.5-1.9)$ & $0.9(0.5-1.8)$ & 0.142 \\
\hline IL-6, mg/L & $36.2(13.4-85.9)$ & $39.8(14.7-94.2)$ & $30.6(12.2-73.8)$ & 0.019 \\
\hline $\mathrm{LDH}, \mathrm{U} / \mathrm{L}$ & $317(243-432)$ & $337(258-450)$ & $293(226-398)$ & $<0.001$ \\
\hline Creatinine, $\mathrm{mg} / \mathrm{L}$ & $0.9(0.7-1.2)$ & $0.9(0.7-1.2)$ & $0.8(0.6-1.1)$ & $<0.001$ \\
\hline
\end{tabular}

Data given as median and interquartile range (IQR) or $n(\%)$; COPD, chronic obstructive pulmonary disease; CRP, C-reactive protein; IL-6, interleukin-6; LDH, lactate dehydrogenase.

\subsection{Relationships between Lymphocyte Counts and Adverse Clinical Outcomes}

Figure 2 depicts absolute lymphocyte counts measured on admission and the relationship with adverse clinical outcomes in COVID-19 patients. The lymphocyte counts on admission were significantly lower in patients who died and those who required ICU admission, invasive mechanical ventilation, or dialysis (all $p<0.001$ ). A Kendall rank correlation analysis demonstrated an inverse correlation between lymphocyte counts and in-hospital mortality (correlation coefficient, $-0.161, p<0.001$ ), ICU admission (correlation coefficient, $-0.134, p<0.001$ ), invasive mechanical ventilation (correlation coefficient, $-0.133, p<0.001$ ), and need for dialysis (correlation coefficient, $-0.068, p<0.001$ ).

A

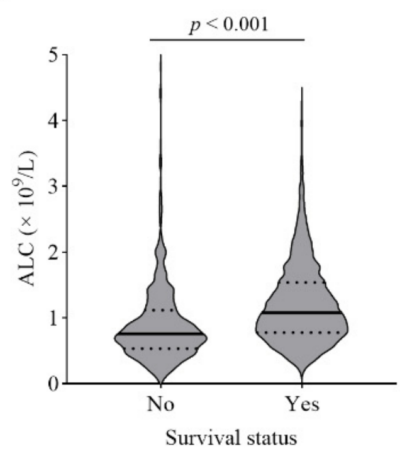

B

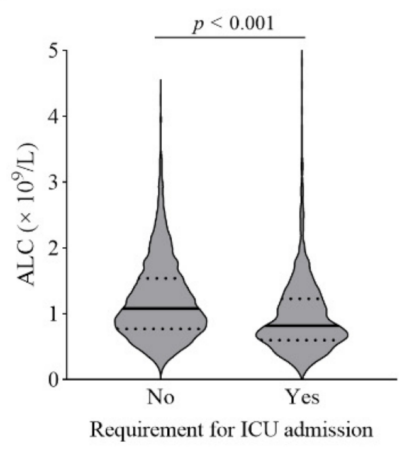

C

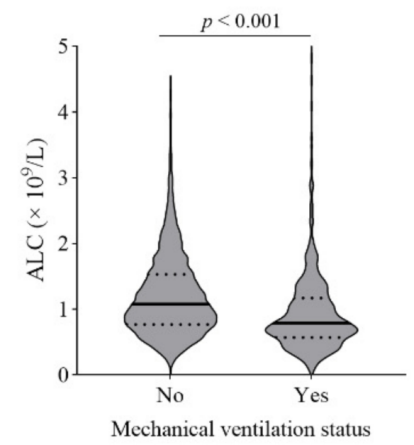

D

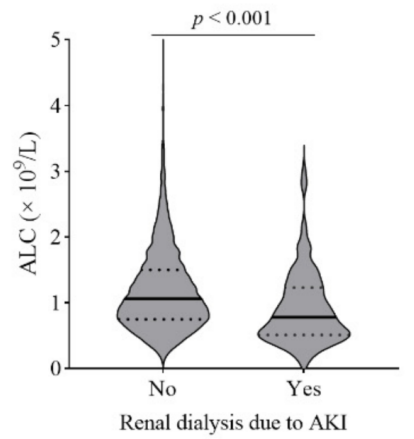

Figure 2. Relationships of ALCs on hospital admission with adverse clinical outcomes in patients with COVID-19. Violin plots of absolute lymphocyte counts (ALCs) showing significantly lower lymphocyte counts in patients who died (A) and those who required ICU admission (B), invasive mechanical ventilation (C), and dialysis (D) than those who were not $(p<0.001$, Mann-Whitney U-test). Solid black lines indicate medians, and dashed black lines represent quartile ranges.

Elevated serum levels of CRP, D-dimer, IL-6, LDH, and creatinine have been associated with disease progression and poor clinical outcomes in COVID-19 patients [9,11]. We analyzed the relationship between lymphocyte counts and the serum levels of CRP, D-dimer, IL-6, LDH, and creatinine. Utilizing Spearman correlation analysis, there was a statistically significant inverse relationship between lymphocyte counts and the serum levels of CRP (correlation coefficient, $-0.130, p<0.001$ ), D-dimer (correlation coefficient, -0.041 , $p=0.014)$, IL-6 (correlation coefficient, $-0.115, p=0.001$ ), LDH (correlation coefficient, 
A

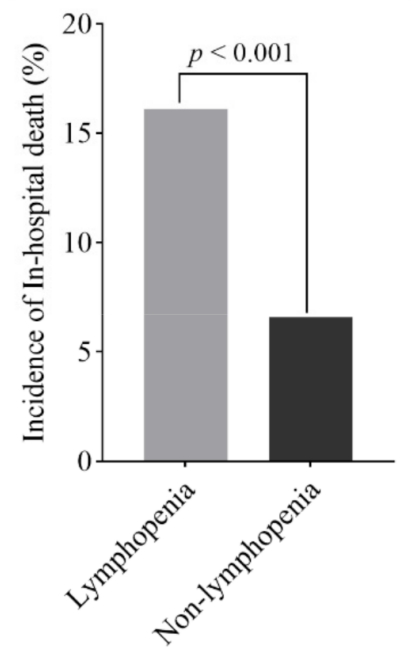

$-0.163, p<0.001$ ), and creatinine (correlation coefficient, $-0.092, p<0.001$ ), respectively, suggesting a clear relationship of a lower lymphocyte count at the time of hospital admission with high levels of CRP, D-dimer, IL-6, LDH, and creatinine in COVID-19 patients.

\subsection{Lymphopenia Is Associated with Higher Incidences of Adverse Clinical Outcomes}

Of the 4485 patients analyzed, 628 (14\%) patients required admission to the ICU, $482(10.7 \%)$ required invasive mechanical ventilation support, $119(2.7 \%)$ required dialysis due to the development of AKI, and 538 (11.9\%) hospitalized patients died during the study period. In comparison to patients who did not have lymphopenia on hospital admission, those with lymphopenia had higher incidences of ICU admission (17.9\% versus 9.5\%, $p<0.001)$, invasive mechanical ventilation $(14.4 \%$ versus $6.5 \%, p<0.001)$, dialysis $(3.4 \%$ versus $1.8 \%, p<0.001)$, and in-hospital mortality $(16.6 \%$ versus $6.6 \%, p<0.001)$, respectively (Figure 3). Patients with lymphopenia also had a longer hospital length of stay compared to those without lymphopenia $(9$ [IQR, 5-16] days versus 7 [IQR, 4-13] days; $p<0.001)$.

B

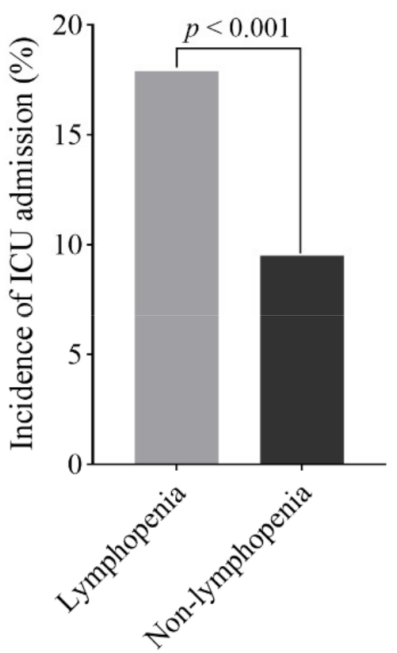

C

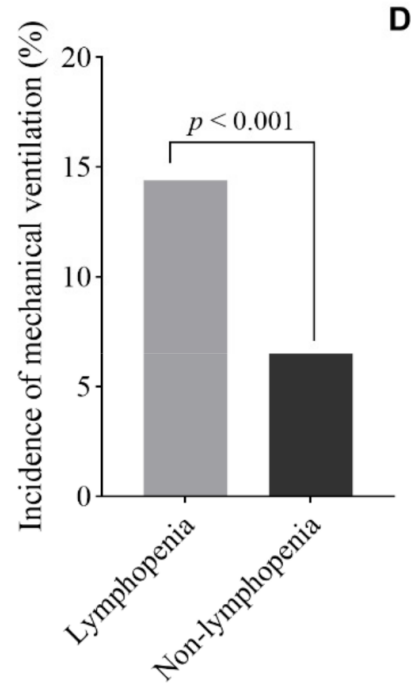

D

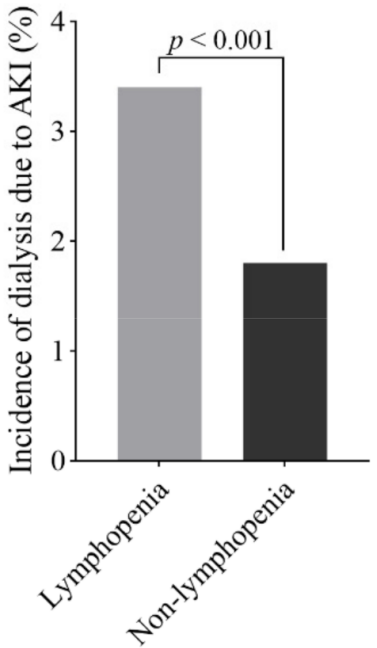

Figure 3. Comparison of the adverse outcomes in patients with or without lymphopenia upon hospital admission. Bar graphs show the incidences of in-hospital death (A), ICU admission (B), invasive mechanical ventilation (C), and dialysis due to AKI (D) in the study population classified into two groups according to their absolute lymphocyte counts at admission. Patients with lymphopenia had higher incidences of adverse outcomes (all $p<0.001)$.

\subsection{Lymphopenia Is an Independent Risk Factor for Adverse Clinical Outcomes}

Logistic regression models were used to measure the association of lymphopenia with adverse clinical outcomes in patients with COVID-19. The unadjusted model revealed a higher risk of adverse outcomes in patients with lymphopenia, of 2.07-fold for ICU admission (OR, 2.07; 95\% CI, 1.73-2.48), 2.37-fold for invasive mechanical ventilation (OR, 2.37; 95\% CI, 1.93-2.92), 1.94-fold for dialysis (OR, 1.94; 95\% CI, 1.32-2.91), and 2.82-fold for in-hospital mortality (OR, 2.82; 95\% CI, 2.30-3.46), compared with patients who did not present with lymphopenia (Figure 4). This pattern of association between lymphopenia and adverse outcomes persisted after adjusting for age and gender, with the adjusted ORs of 1.84 (95\% CI, 1.54-2.22) for ICU admission, 2.09 (95\% CI, 1.69-2.59) for invasive mechanical ventilation, 1.72 (95\% CI, 1.16-2.60) for dialysis, and 2.18 (95\% CI, 1.76-2.70) for in-hospital mortality, respectively (Figure 4). We then created a multivariable logistic regression model that included age, gender, race, and history of hypertension, diabetes, COPD, chronic kidney disease, coronary artery disease, malignancy, obesity, and smoking as covariates. After adjustment for these variables, the presence of lymphopenia was still found to be associated with the risks of adverse outcomes, with multivariable-adjusted ORs of 1.86 (95\% CI, 1.55-2.25) for ICU admission, 2.09 (95\% CI, 1.69-2.59) for invasive 
mechanical ventilation, 1.77 (95\% CI, 1.19-2.68) for dialysis, and 2.19 (95\% CI, 1.76-2.72) for in-hospital mortality, respectively, in comparison with individuals who did not have lymphopenia (Figure 4).

A

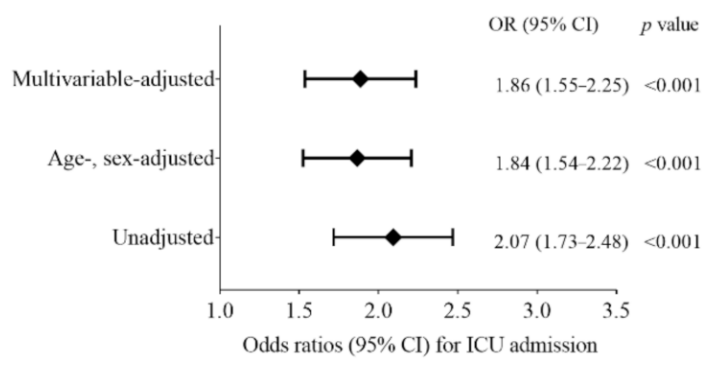

C

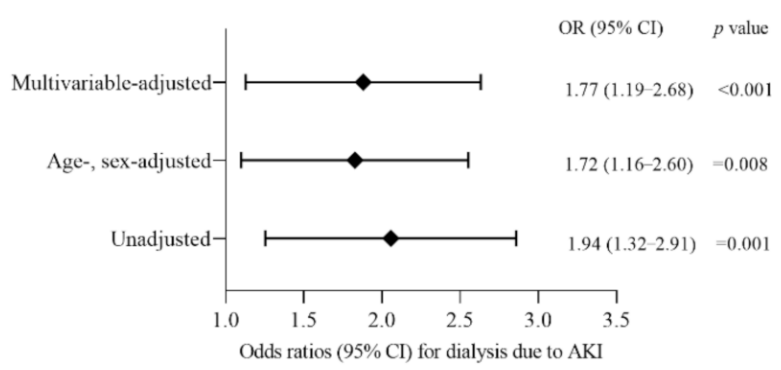

B

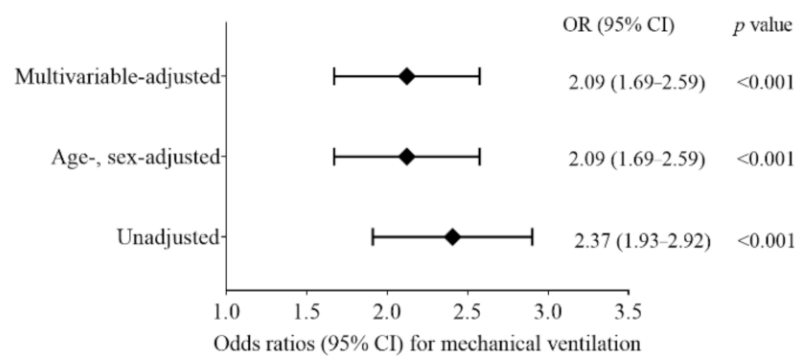

D

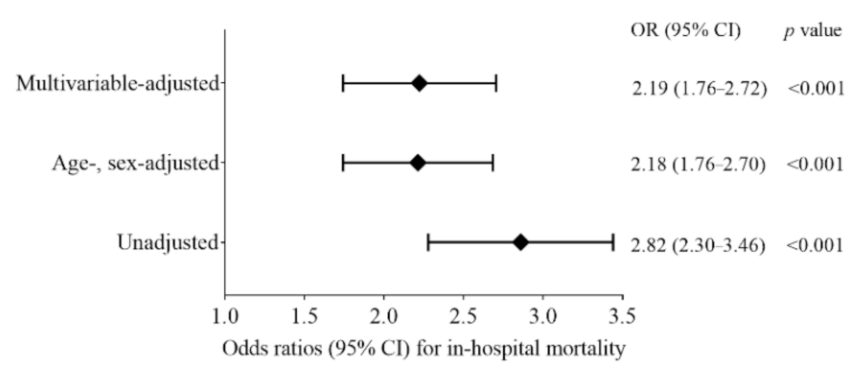

Figure 4. Forest plots showing association of lymphopenia with risks of adverse clinical outcomes in patients with COVID-19. Independent effects of lymphopenia (ALC $<1.1 \times 10^{9} / \mathrm{L}$ ) on the risks of ICU admission (A), invasive mechanical ventilation (B), dialysis due to AKI (C), and inhospital mortality (D) during hospitalization were analyzed using logistic regression models and compared to individuals with ALC $\geq 1.1 \times 10^{9} / \mathrm{L}$. Results were reported as odds ratios (ORs) with 95\% confidence intervals (CIs), adjusted for age-, sex-, and multi-variables including age, sex, race, diabetes, hypertension, obesity, smoking, COPD, CKD, CHD, and malignancy. ICU, intensive care unit; AKI, acute kidney injury.

\section{5. "Dose-Response" Relationships between Lymphopenia and Adverse Outcomes}

The spline curves in Figure 5 illustrates the "dose-response" relationship between continuous changes in lymphocyte counts and changes in ORs for the risks of adverse outcomes, assessed using the RCS models adjusted for covariates including age, gender, race, and history of hypertension, diabetes, COPD, chronic kidney disease, coronary artery disease, malignancy, obesity, and smoking. When ALC levels were below $1.1 \times 10^{9} / \mathrm{L}$ (lymphopenia), a negative nonlinear dose-response relationship was observed, with the highest ORs for adverse outcomes seen in patients with the lowest values of ALC. There was a sharp increase in the values of multivariable-adjusted ORs for in-hospital death, ICU admission, mechanical ventilation, and dialysis, with a decreasing of ALC, ultimately reaching corresponding ORs of 5.22, 4.44, 5.18, and 8.05, respectively at the lowest lymphocyte counts of $0.1 \times 10^{9} / \mathrm{L}$. When the ALC values were greater than or equal to $1.1 \times 10^{9} / \mathrm{L}$, multivariable-adjusted ORs for adverse outcomes did not differ from 1.0-reflecting the absence of a significant association between ALC and the risk of adverse outcomes. 
A

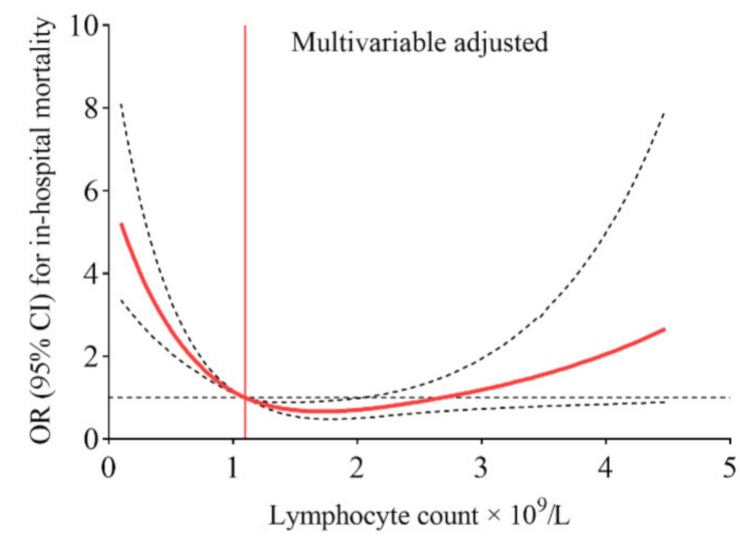

C

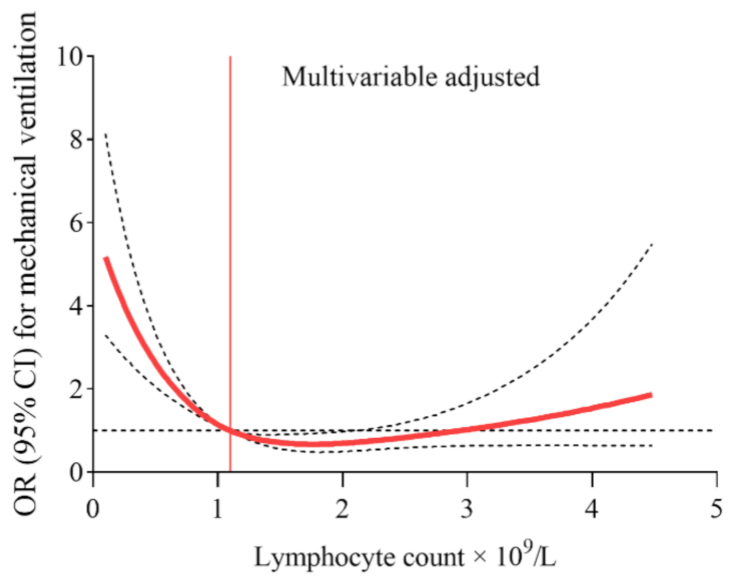

B

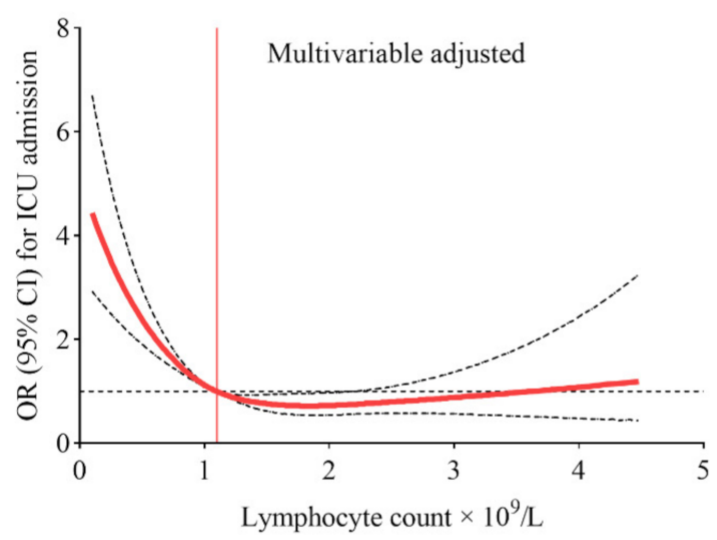

D

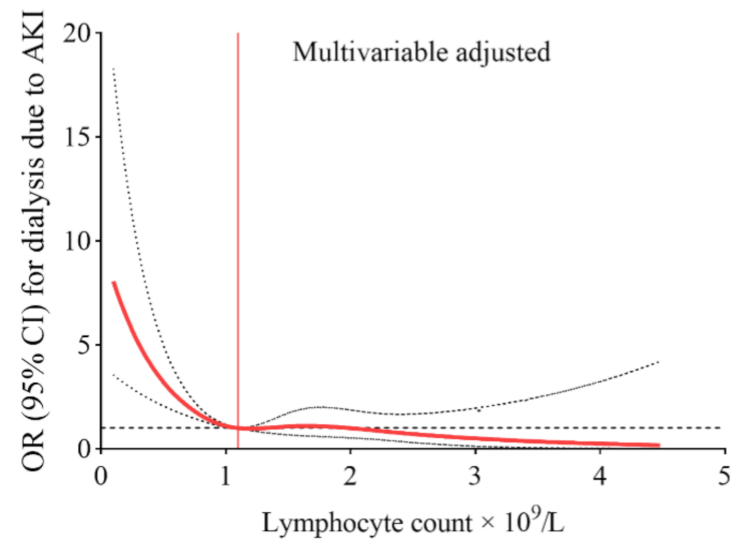

Figure 5. Dose-response association of absolute lymphocyte count and risk of the outcomes of interest in patients with COVID-19. Restricted cubic splines were generated using logistic regression models, and the lymphocyte value of $1.1 \times 10^{9} / \mathrm{L}$ was set as reference (denoted by red vertical lines) for the continuous model adjusted to age, gender, race, and history of hypertension, diabetes, chronic obstructive pulmonary disease, chronic kidney disease, coronary artery disease, malignancy, obesity, and smoking. Solid red curves are ORs for the outcomes of interest (A-D), and dashed black lines indicate $95 \%$ confidence intervals based on fitting of cubic splines to risk estimates obtained using logistic regression.

\subsection{Lymphopenia Predicts the Risk of Adverse Clinical Outcomes in COVID-19 Patients}

To explore lymphopenia as a tool to predict patients' risk of adverse outcomes due to COVID-19 infection, receiver operating characteristic (ROC) curves and areas under ROC curves (AUCs) were calculated. Figure 6 (black curves) shows the ROC curves of lymphopenia for predicting risks of in-hospital mortality, ICU admission, invasive mechanical ventilation, and dialysis in COVID-19 patients, with the corresponding AUCs of 0.62 (95\% CI, 0.59-0.64), 0.59 (95\% CI, 0.56-0.60), 0.60 (95\% CI, 0.58-0.63), and 0.58 (95\% CI, 0.53-0.63), respectively. 
A

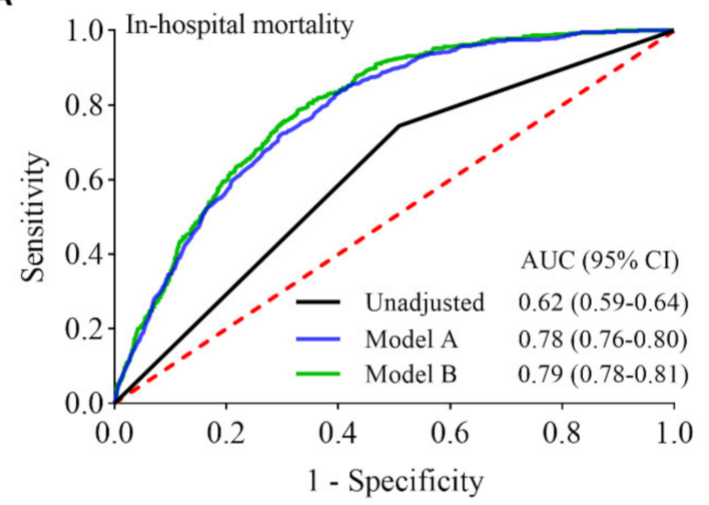

C

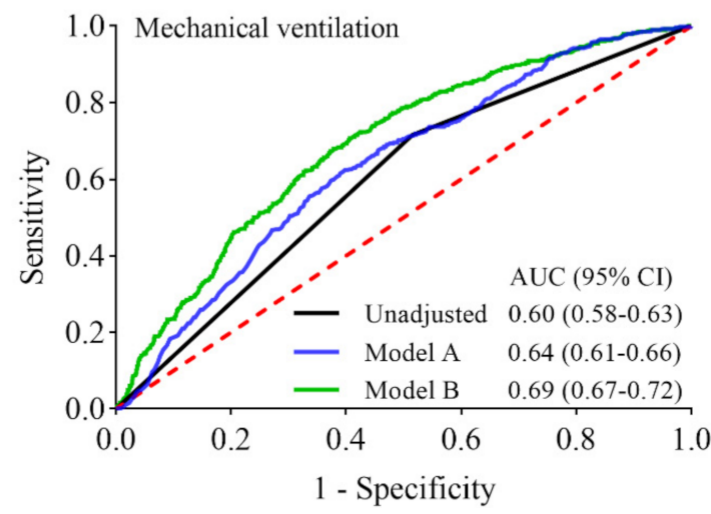

B

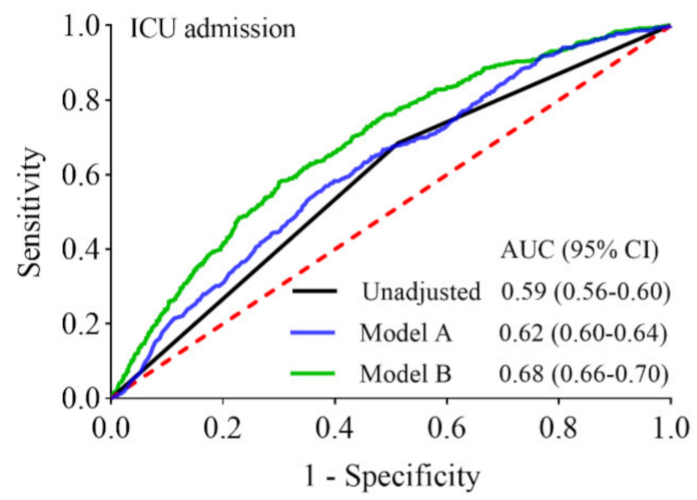

D

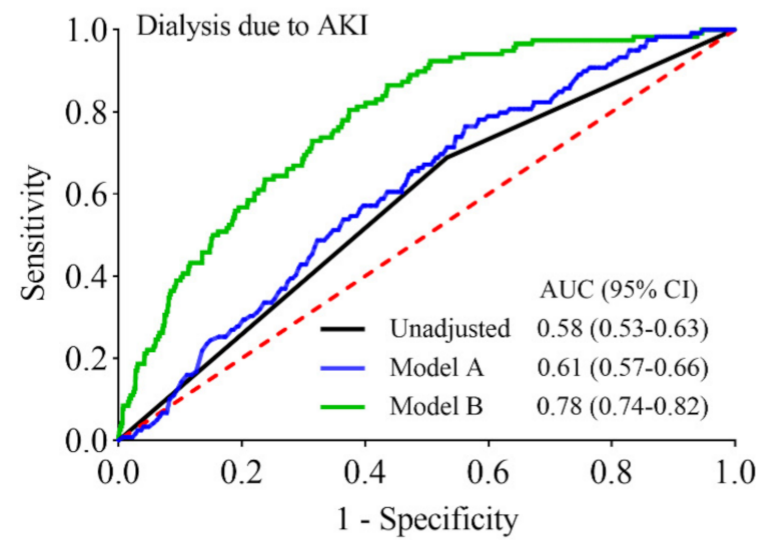

Figure 6. ROC curves of lymphopenia for predicting risks of the outcomes of interest. The areas under the ROC curves (AUCs) are values for estimating incidences of the outcomes of interest (A-D) through the use of logistic regression function calculated from unadjusted lymphopenia, model A, and model B, respectively. Model A, adjusted for age and gender; Model B, adjusted for age, gender, race, hypertension, diabetes, COPD, chronic kidney disease, coronary artery disease, malignancy, obesity, and smoking. Lymphopenia have the largest AUCs (areas under the green curves) to predict individual risk of adverse clinical outcomes with the incorporation of the known clinical risk factors. AKI, acute kidney injury; CI, confidence interval.

When taking age and gender into account, the predictive power of the combination of lymphopenia with age and gender in the risk model (model A) is superior to lymphopenia alone as a predictor of in-hospital mortality, as illustrated by the gap between two AUCs of 95\% CIs (Figure 6A, blue and black curves; $p<0.001$ ). There was no significant difference in the AUCs between lymphopenia alone and the combination of lymphopenia with age and gender in the risk models for ICU admission, invasive mechanical ventilation, and the need for dialysis due to AKI (Figure 6B-D, blue and black curves; $p=0.052$, $p=0.103$, and $p=0.327$, respectively). Thus, the predictive power of the combination of lymphopenia with age and gender is similar to lymphopenia alone for predicting the risks of ICU admission, invasive mechanical ventilation, and dialysis. Utilizing an extensive combination of additional variables (age, gender, race, hypertension, diabetes, COPD, chronic kidney disease, coronary artery disease, malignancy, obesity, and smoking) together with the presence of lymphopenia in the predictive risk model (model B) enhances the predictive powers for the corresponding adverse outcomes, as demonstrated by the AUC values of 0.79 (95\% CI, 0.78-0.81), 0.68 (95\% CI, 0.66-0.70), 0.69 (95\% CI, 0.67-0.72), 0.78 (95\% CI, 0.74-0.82), respectively (Figure 6, green and black curves; all $p<0.001$ ). 


\section{Discussion}

COVID-19 is a highly infectious disease with significant morbidity and mortality. Gaining further insight into models predicting adverse clinical outcomes could help identify high-risk patients and prioritize workflow to optimize patient care. In this retrospective, population-based study of 4485 individuals, lymphopenia, defined as blood lymphocytes $<1.1 \times 10^{9} / \mathrm{L}$, occurred in $53.7 \%$ of patients with COVID-19 at the time of admission. Patients with lymphopenia tended to be much older, male, and had higher rates of comorbid diseases than their non-lymphopenic counterparts. The lymphocyte counts were inversely associated with serum levels of CRP, IL-6, D-dimer, LDH, and creatinine. Lymphopenia was found to be an independent risk marker for ICU admission, invasive mechanical ventilation, dialysis, and in-hospital mortality. Moreover, a negative nonlinear dose-response relationship between lymphopenia and adverse outcomes was observed, with higher risks of adverse outcomes seen in patients with the lowest values of lymphocyte counts in patients with lymphopenia. Furthermore, the prognostic power of lymphopenia for adverse clinical outcomes were further strengthened by the incorporation of age, gender, race, and comorbidities.

Lymphopenia has become a well-recognized risk marker for the severity of COVID-19 [21-23,26]. Lymphopenia is associated with a higher prevalence of the known risk factors of COVID-19 such as older age, male gender, hypertension, COPD, chronic kidney disease, coronary heart disease, and obesity [7,27-29]. This observation is welldocumented in our data, showing a significantly higher comorbidity profile in the study population. Lymphopenia is also correlated with a hyper-inflammatory response [30], characterized by high serum levels of CRP, IL-6, D-dimer, and LDH, which is known to be related to the severity and worse outcomes of COVID-19 [8,9,31]. Data in our study supports these findings. We observed that the group of COVID 19 patients with lymphopenia had higher serum levels of pro-inflammatory mediators and higher rates of ICU admission, mechanical ventilation, dialysis due to AKI, and mortality, similarly seen in other COVID-19 studies [30,32,33]. Physiologically, a plausible mechanism linking lymphopenia to poor clinical outcomes specifically involves $\mathrm{T}$ lymphocytes. A higher total $\mathrm{T}$ cell count, including both CD4+ and CD8+, has been shown to be a predictor of a less severe disease and a more favorable clinical outcome in patients with COVID-19 [34]. In The Lancet HIV, two studies demonstrated worse outcomes in HIV patients with CD4 counts less than 200 cells per $\mu \mathrm{L}[35,36]$. Overall, $70-80 \%$ of circulating lymphocytes are $\mathrm{T}$ lymphocytes and the underlying cause of lymphopenia in severe cases of COVID-19 has previously been attributed to apoptosis of T lymphocytes [37-39]. Mechanistically, SARS-CoV-2 infection can directly infect lymphocytes and induce cell death [40]. The generation of excessive pro-inflammatory cytokines, such as TNF- $\alpha$ and IL-6, can cause robust apoptosis of lymphocytes [41]. The apoptotic T lymphocytes can release the cognate ligand for Fas (FasL) that induces excessive apoptosis of epithelial cells and inflammation, resulting in pulmonary fibrosis [42,43]. Furthermore, SARS-CoV-2 not only diminishes the number of host's lymphocytes but also induces an exhaustion of effector T cells and overproduction of pro-inflammatory cytokines, which in turn contributes to the disease progression and unfavorable outcomes. This observation is supported by the fact that administration of Tocilizumab, a specific anti-IL-6 monoclonal antibody, could increase the number of peripheral lymphocytes and reduce the pulmonary opacification in severe COVID-19 [44]. Therefore, preventing lymphopenia or restoring lymphocyte counts could represent a viable therapeutic opportunity in patients with severe COVID-19 [45-47]. Additional study is needed to characterize apoptosis in lymphocyte subsets and to explore the lines of cause and effect that may contribute to disease progression in COVID-19 patients.

Previous studies have explored lymphopenia in predicting clinical outcomes in patients with COVID-19 $[23,26,33,48]$. Although the definition of lymphopenia varied among studies, lymphopenia, defined as a lymphocyte count $<1.0 \times 10^{9} / \mathrm{L}$ on admission, has been associated with the severity of disease and mortality from COVID-19 [33,48,49]. A subgroup analysis using a lymphocyte count $<1.5 \times 10^{9} / \mathrm{L}$ to define lymphopenia showed 
a 3-fold increased risk of severe COVID-19 infection [26]. In this study, we set a cut-off point of $<1.1 \times 10^{9} / \mathrm{L}$ to define lymphopenia based on 4 prior studies using this cutoff point, previously demonstrating a consistent relationship between lymphopenia and the severity of COVID-19 [50-52]. We found that lymphopenia is relatively common with $53.7 \%$ of patients having lymphopenia on presentation-comparable prevalence to that of large-sized cohort studies from the United States [53,54] and China [55]. Lymphopenia was consistently found to be independently associated with adverse clinical outcomes, namely ICU admission, the use of mechanical ventilation, dialysis and in-hospital mortality. These data are consistent with prior findings showing an increased risk for worse outcomes among patients with lymphopenia [21,33,51,55-58]. Our study provides additional data about a negative nonlinear dose-response relationship between lymphopenia and risks for the adverse outcomes, highlighting a higher risk profile for adverse outcomes in patients with the lowest values of ALC at the time of admission [21]. The ROC analyses reveal that utilizing an extensive combination of additional variables together with the presence of lymphopenia in the predictive risk model enhances the predictive ability of the model for adverse outcomes.

The present study is limited by being a single center, retrospective cohort study. Selection bias and other limitations may exist-and all potential confounders may have not been included in the multivariable adjusted analyses. In this study, we aimed to focus on admission findings as predictive markers for adverse outcomes, hence our multivariable adjusted analyses did not include the type and timing of treatments as variables, which may impact the clinical outcomes in COVID-19 patients. For instance, corticosteroid use in patients with lymphocyte counts $\leq 0.82 \times 10^{9} / \mathrm{L}$ was associated with a significantly reduced risk of all-cause mortality, whereas no significant difference was found in patients with lymphocytes $>0.82 \times 10^{9} / \mathrm{L}$ receiving corticosteroids [59]. A meta-analysis of 44 studies comprising 20,197 patients demonstrates a beneficial effect of corticosteroids on short-term mortality and a reduction in the need for mechanical ventilation [60]. In addition, ALC was assessed on admission to hospital only. We did not evaluate the impact of dynamic changes of lymphocyte counts on clinical outcomes. Additionally, we did not measure specific lymphocyte subsets; thus, we do not know the degree to which lymphopenia was pronounced in each subset of lymphocytes, which represents important data that may provide further insight into disease severity and outcomes of COVID-19 hospitalized patients. Despite these limitations, our findings were internally consistent and in agreement with the previous published studies $[21,22,26,33,49]$.

\section{Conclusions}

Lymphopenia, defined as ALC $<1.1 \times 10^{9} / \mathrm{L}$, is an independent risk factor associated with adverse outcomes in hospitalized COVID-19 patients. Patients with lymphopenia with the lowest ALC values are more likely to have a poor prognosis. Early recognition of this immunological phenotype could assist in the timely recognition and prognostication of severe COVID-19 patients. As lymphocytes are a pivotal component of the host immune responses to SARS-CoV-2 infection, our results highlight that targeting lymphocytes could be a promising strategy in the treatment of SARS-CoV-2 virus infection.

Author Contributions: Conceptualization, J.N. and A.S.; methodology, J.N.; validation, J.N., C.S. and A.S.; formal analysis, J.N.; data curation, C.S.; writing—original draft preparation, J.N.; writingreview and editing, J.N., C.S., D.M., A.V. and A.S. All authors have read and agreed to the published version of the manuscript.

Funding: This research received no external funding.

Institutional Review Board Statement: The study was conducted in accordance with the Declaration of Helsinki, and approved by the Institutional Review Board of Memorial Healthcare System (protocol No. MHS.2020.065) for studies involving humans.

Informed Consent Statement: Patient consent was waived as this was a retrospective analysis of existing data. 
Data Availability Statement: Data can be obtained from the corresponding author upon reasonable request.

Acknowledgments: The authors want to thank the IT Department of the Memorial Healthcare System for kindly providing the original data used in the study.

Conflicts of Interest: The authors declare no conflict of interest.

\section{References}

1. Hu, B.; Guo, H.; Zhou, P.; Shi, Z.L. Characteristics of SARS-CoV-2 and COVID-19. Nat. Rev. Microbiol. 2021, 19, 141-154. [CrossRef]

2. Wu, Z.; McGoogan, J.M. Characteristics of and important lessons from the coronavirus disease 2019 (COVID-19) outbreak in China: Summary of a report of 72314 cases from the Chinese Center for Disease Control and Prevention. JAMA 2020, 323, 1239-1242. [CrossRef] [PubMed]

3. Biolè, C.; Bianco, M.; Núñez-Gil, I.J.; Cerrato, E.; Spirito, A.; Roubin, S.R.; Viana-Llamas, M.C.; Gonzalez, A.; Castro-Mejía, A.F.; Eid, C.M.; et al. Gender Differences in the Presentation and Outcomes of Hospitalized Patients With COVID-19. J. Hosp. Med. 2021, 16, 349-352. [CrossRef] [PubMed]

4. Núñez-Gil, I.J.; Fernández-Pérez, C.; Estrada, V.; Becerra-Muñoz, V.M.; El-Battrawy, I.; Uribarri, A.; Fernández-Rozas, I.; Feltes, G.; Viana-Llamas, M.C.; Trabattoni, D.; et al. Mortality risk assessment in Spain and Italy, insights of the HOPE COVID-19 registry. Intern. Emerg. Med. 2020, 16, 957-966. [CrossRef] [PubMed]

5. Booth, A.; Reed, A.B.; Ponzo, S.; Yassaee, A.; Aral, M.; Plans, D.; Labrique, A.; Mohan, D. Population risk factors for severe disease and mortality in COVID-19: A global systematic review and meta-analysis. PLoS ONE 2021, 16, e0247461. [CrossRef] [PubMed]

6. Mallow, P.J.; Belk, K.W.; Topmiller, M.; Hooker, E.A. Outcomes of Hospitalized COVID-19 Patients by Risk Factors: Results from a United States Hospital Claims Database. J. Health Econ. Outcomes Res. 2020, 7, 165-175. [CrossRef] [PubMed]

7. Hanif, M.; Haider, M.A.; Xi, Q.; Ali, M.J.; Ahmed, M.U. A Review of the Risk Factors Associated with Poor Outcomes in Patients with Coronavirus Disease 2019. Cureus 2020, 12. [CrossRef] [PubMed]

8. Malik, P.; Patel, U.; Mehta, D.; Patel, N.; Kelkar, R.; Akrmah, M.; Gabrilove, J.L.; Sacks, H. Biomarkers and outcomes of COVID-19 hospitalisations: Systematic review and meta-analysis. BMJ Evid.-Based Med. 2020, 26, 107-108. [CrossRef]

9. Ponti, G.; Maccaferri, M.; Ruini, C.; Tomasi, A.; Ozben, T. Biomarkers associated with COVID-19 disease progression. Crit. Rev. Clin. Lab. Sci. 2020, 57, 389-399. [CrossRef]

10. Wynants, L.; Van Calster, B.; Collins, G.S.; Riley, R.D.; Heinze, G.; Schuit, E.; Bonten, M.M.J.; Dahly, D.L.; Damen, J.A.; Debray, T.P.A.; et al. Prediction models for diagnosis and prognosis of COVID-19: Systematic review and critical appraisal. BMJ 2020, 369, m1328. [CrossRef]

11. Elshazli, R.M.; Toraih, E.A.; Elgaml, A.; El-Mowafy, M.; El-Mesery, M.; Amin, M.N.; Hussein, M.H.; Killackey, M.T.; Fawzy, M.S.; Kandil, E. Diagnostic and prognostic value of hematological and immunological markers in COVID-19 infection: A meta-analysis of 6320 patients. PLoS ONE 2020, 15, e0238160. [CrossRef]

12. Koyasu, S.; Moro, K. Role of Innate Lymphocytes in Infection and Inflammation. Front. Immunol. 2012, 3, 101. [CrossRef]

13. Okada, H.; Kobune, F.; Sato, T.A.; Kohama, T.; Takeuchi, Y.; Abe, T.; Takayama, N.; Tsuchiya, T.; Tashiro, M. Extensive lymphopenia due to apoptosis of uninfected lymphocytes in acute measles patients. Arch. Virol. 2000, 145, 905-920. [CrossRef]

14. Cheng, Y.; Zhao, H.; Song, P.; Zhang, Z.; Chen, J.; Zhou, Y.-H. Dynamic changes of lymphocyte counts in adult patients with severe pandemic H1N1 influenza A. J. Infect. Public Health 2019, 12, 878-883. [CrossRef]

15. Gooskens, J.; Jonges, M.; Claas, E.C.J.; Meijer, A.; Kroes, A.C.M. Prolonged Influenza Virus Infection during Lymphocytopenia and Frequent Detection of Drug-Resistant Viruses. J. Infect. Dis. 2009, 199, 1435-1441. [CrossRef]

16. Bellelli, V.; D’Ettorre, G.; Celani, L.; Borrazzo, C.; Ceccarelli, G.; Venditti, M. Clinical significance of lymphocytopenia in patients hospitalized with pneumonia caused by influenza virus. Crit. Care 2019, 23, 330. [CrossRef]

17. Moon, S.W.; Leem, A.Y.; Kim, Y.S.; Lee, J.-H.; Kim, T.-H.; Oh, Y.-M.; Shin, H.; Chang, J.; Jung, J.Y. Low serum lymphocyte level is associated with poor exercise capacity and quality of life in chronic obstructive pulmonary disease. Sci. Rep. 2020, 10, 11700. [CrossRef]

18. Ommen, S.R.; Hodge, D.O.; Rodeheffer, R.J.; McGregor, C.G.; Thomson, S.P.; Gibbons, R.J. Predictive power of the relative lymphocyte concentration in patients with advanced heart failure. Circulation 1998, 97, 19-22. [CrossRef]

19. Vaduganathan, M.; Ambrosy, A.P.; Greene, S.J.; Mentz, R.J.; Subacius, H.P.; Maggioni, A.P.; Swedberg, K.; Nodari, S.; Zannad, F.; Konstam, M.A.; et al. Predictive value of low relative lymphocyte count in patients hospitalized for heart failure with reduced ejection fraction: Insights from the EVEREST trial. Circ. Heart Fail. 2012, 5, 750-758. [CrossRef]

20. Ray-Coquard, I.; Cropet, C.; Van Glabbeke, M.; Sebban, C.; Le Cesne, A.; Judson, I.; Tredan, O.; Verweij, J.; Biron, P.; Labidi, I.; et al. Lymphopenia as a prognostic factor for overall survival in advanced carcinomas, sarcomas, and lymphomas. Cancer Res. 2009, 69, 5383-5391. [CrossRef]

21. Huang, I.; Pranata, R. Lymphopenia in severe coronavirus disease-2019 (COVID-19): Systematic review and meta-analysis. J. Intensive Care 2020, 8, 36. [CrossRef] [PubMed]

22. Illg, Z.; Muller, G.; Mueller, M.; Nippert, J.; Allen, B. Analysis of absolute lymphocyte count in patients with COVID-19. Am. J. Emerg. Med. 2021, 46, 16-19. [CrossRef] [PubMed] 
23. Henry, B.; Cheruiyot, I.; Vikse, J.; Mutua, V.; Kipkorir, V.; Benoit, J.; Plebani, M.; Bragazzi, N.; Lippi, G. Lymphopenia and neutrophilia at admission predicts severity and mortality in patients with COVID-19: A meta-analysis. Acta Biomed. 2020, 91, e2020008. [CrossRef] [PubMed]

24. Marrie, R.A.; Dawson, N.V.; Garland, A. Quantile regression and restricted cubic splines are useful for exploring relationships between continuous variables. J. Clin. Epidemiol. 2009, 62, 511-517. [CrossRef] [PubMed]

25. DeLong, E.R.; DeLong, D.M.; Clarke-Pearson, D.L. Comparing the areas under two or more correlated receiver operating characteristic curves: A nonparametric approach. Biometrics 1988, 44, 837-845. [CrossRef]

26. Zhao, Q.; Meng, M.; Kumar, R.; Wu, Y.; Huang, J.; Deng, Y.; Weng, Z.; Yang, L. Lymphopenia is associated with severe coronavirus disease 2019 (COVID-19) infections: A systemic review and meta-analysis. Int. J. Infect. Dis. 2020, 96, 131-135. [CrossRef]

27. Gao, Y.-D.; Ding, M.; Dong, X.; Zhang, J.-J.; Azkur, A.K.; Azkur, D.; Gan, H.; Sun, Y.-L.; Fu, W.; Li, W.; et al. Risk factors for severe and critically ill COVID-19 patients: A review. Allergy 2021, 76, 428-455. [CrossRef]

28. Malik, P.; Patel, U.; Patel, K.; Martin, M.; Shah, C.; Mehta, D.; Malik, F.A.; Sharma, A. Obesity a predictor of outcomes of COVID-19 hospitalized patients-A systematic review and meta-analysis. J. Med. Virol. 2021, 93, 1188-1193. [CrossRef]

29. Patel, U.; Malik, P.; Shah, D.; Patel, A.; Dhamoon, M.; Jani, V. Pre-existing cerebrovascular disease and poor outcomes of COVID-19 hospitalized patients: A meta-analysis. J. Neurol. 2020, 268, 240-247. [CrossRef]

30. Diao, B.; Wang, C.; Tan, Y.; Chen, X.; Liu, Y.; Ning, L.; Chen, L.; Li, M.; Liu, Y.; Wang, G.; et al. Reduction and Functional Exhaustion of T Cells in Patients with Coronavirus Disease 2019 (COVID-19). Front. Immunol. 2020, 11, 827. [CrossRef]

31. Soraya, G.V.; Ulhaq, Z.S. Crucial laboratory parameters in COVID-19 diagnosis and prognosis: An updated meta-analysis. Med. Clin. 2020, 155, 143-151. [CrossRef]

32. Bordoni, V.; Sacchi, A.; Cimini, E.; Notari, S.; Grassi, G.; Tartaglia, E.; Casetti, R.; Giancola, M.L.; Bevilacqua, N.; Maeurer, M.; et al An inflammatory profile correlates with decreased frequency of cytotoxic cells in coronavirus disease 2019. Clin. Infect. Dis. 2020, 71, 2272-2275. [CrossRef]

33. Huang, G.; Kovalic, A.J.; Graber, C.J. Prognostic Value of Leukocytosis and Lymphopenia for Coronavirus Disease Severity Emerg. Infect. Dis. 2020, 26, 1839-1841. [CrossRef]

34. Liu, Z.; Long, W.; Tu, M.; Chen, S.; Huang, Y.; Wang, S.; Zhou, W.; Chen, D.; Zhou, L.; Wang, M.; et al. Lymphocyte subset (CD4+, CD8+) counts reflect the severity of infection and predict the clinical outcomes in patients with COVID-19. J. Infect. 2020, 81, 318-356. [CrossRef]

35. Nomah, D.K.; Reyes-Urueña, J.; Diaz, Y.; Moreno, S.; Aceiton, J.; Bruguera, A.; Vivanco-Hidalgo, R.M.; Llibre, J.M.; Domingo, P.; Falcó, V.; et al. Sociodemographic, clinical, and immunological factors associated with SARS-CoV-2 diagnosis and severe COVID-19 outcomes in people living with HIV: A retrospective cohort study. Lancet HIV 2021, 8, e701-e710. [CrossRef]

36. Yang, X.; Sun, J.; Patel, R.C.; Zhang, J.; Guo, S.; Zheng, Q.; Olex, A.L.; Olatosi, B.; Weissman, S.B.; Islam, J.Y.; et al. Associations between HIV infection and clinical spectrum of COVID-19: A population level analysis based on US National COVID Cohort Collaborative (N3C) data. Lancet HIV 2021, 8, e690-e700. [CrossRef]

37. Qu, R.; Ling, Y.; Zhang, Y.H.; Wei, L.Y.; Chen, X.; Li, X.M.; Liu, X.Y.; Liu, H.M.; Guo, Z.; Ren, H.; et al. Platelet-to-lymphocyte ratio is associated with prognosis in patients with coronavirus disease-19. J. Med. Virol. 2020, 92, 1533-1541. [CrossRef]

38. Cizmecioglu, A.; Akay Cizmecioglu, H.; Goktepe, M.H.; Emsen, A.; Korkmaz, C.; Esenkaya Tasbent, F.; Colkesen, F.; Artac, H. Apoptosis-induced T-cell lymphopenia is related to COVID-19 severity. J. Med. Virol. 2021, 93, 2867-2874. [CrossRef]

39. Urra, J.M.; Cabrera, C.M.; Porras, L.; Rodenas, I. Selective CD8 cell reduction by SARS-CoV-2 is associated with a worse prognosis and systemic inflammation in COVID-19 patients. Clin. Immunol. 2020, 217, 108486. [CrossRef]

40. Ren, Y.; Shu, T.; Wu, D.; Mu, J.; Wang, C.; Huang, M.; Han, Y.; Zhang, X.Y.; Zhou, W.; Qiu, Y.; et al. The ORF3a protein of SARS-CoV-2 induces apoptosis in cells. Cell. Mol. Immunol. 2020, 17, 881-883. [CrossRef]

41. Liao, Y.-C.; Liang, W.-G.; Chen, F.-W.; Hsu, J.-H.; Yang, J.-J.; Chang, M.-S. IL-19 Induces Production of IL-6 and TNF- $\alpha$ and Results in Cell Apoptosis Through TNF- $\alpha$. J. Immunol. 2002, 169, 4288-4297. [CrossRef] [PubMed]

42. Hagimoto, N.; Kuwano, K.; Miyazaki, H.; Kunitake, R.; Fujita, M.; Kawasaki, M.; Kaneko, Y.; Hara, N. Induction of Apoptosis and Pulmonary Fibrosis in Mice in Response to Ligation of Fas Antigen. Am. J. Respir. Cell Mol. Biol. 1997, 17, 272-278. [CrossRef] [PubMed]

43. Drakopanagiotakis, F.; Xifteri, A.; Polychronopoulos, V.; Bouros, D. Apoptosis in lung injury and fibrosis. Eur. Respir. J. 2008, 32, 1631-1638. [CrossRef] [PubMed]

44. Xu, X.; Han, M.; Li, T.; Sun, W.; Wang, D.; Fu, B.; Zhou, Y.; Zheng, X.; Yang, Y.; Li, X.; et al. Effective treatment of severe COVID-19 patients with tocilizumab. Proc. Natl. Acad. Sci. USA 2020, 117, 10970-10975. [CrossRef]

45. Yang, L.; Liu, S.; Liu, J.; Zhang, Z.; Wan, X.; Huang, B.; Chen, Y.; Zhang, Y. COVID-19: Immunopathogenesis and Immunotherapeutics. Signal Transduct. Target. Ther. 2020, 5, 128. [CrossRef]

46. Fathi, N.; Rezaei, N. Lymphopenia in COVID-19: Therapeutic opportunities. Cell Biol. Int. 2020, 44, 1792-1797. [CrossRef]

47. Delshad, M.; Tavakolinia, N.; Pourbagheri-Sigaroodi, A.; Safaroghli-Azar, A.; Bagheri, N.; Bashash, D. The contributory role of lymphocyte subsets, pathophysiology of lymphopenia and its implication as prognostic and therapeutic opportunity in COVID-19. Int. Immunopharmacol. 2021, 95, 107586. [CrossRef]

48. Liu, J.; Li, H.; Luo, M.; Liu, J.; Wu, L.; Lin, X.; Li, R.; Wang, Z.; Zhong, H.; Zheng, W.; et al. Lymphopenia predicted illness severity and recovery in patients with COVID-19: A single-center, retrospective study. PLoS ONE 2020, 15, e0241659. [CrossRef] 
49. Lee, J.; Park, S.S.; Kim, T.Y.; Lee, D.G.; Kim, D.W. Lymphopenia as a biological predictor of outcomes in COVID-19 patients: A nationwide cohort study. Cancers 2021, 13, 471. [CrossRef]

50. Liu, W.; Tao, Z.-W.; Wang, L.; Yuan, M.-L.; Liu, K.; Zhou, L.; Wei, S.; Deng, Y.; Liu, J.; Liu, H.-G.; et al. Analysis of factors associated with disease outcomes in hospitalized patients with 2019 novel coronavirus disease. Chin. Med. J. 2020, 133, 1032-1038. [CrossRef]

51. Yang, X.; Yu, Y.; Xu, J.; Shu, H.; Xia, J.; Liu, H.; Wu, Y.; Zhang, L.; Yu, Z.; Fang, M.; et al. Clinical course and outcomes of critically ill patients with SARS-CoV-2 pneumonia in Wuhan, China: A single-centered, retrospective, observational study. Lancet Respir. Med. 2020, 8, 475-481. [CrossRef]

52. Zhang, J.J.; Dong, X.; Cao, Y.Y.; Yuan, Y.D.; Yang, Y.B.; Yan, Y.Q.; Akdis, C.A.; Gao, Y.D. Clinical characteristics of 140 patients infected with SARS-CoV-2 in Wuhan, China. Allergy 2020, 75, 1730-1741. [CrossRef]

53. Richardson, S.; Hirsch, J.S.; Narasimhan, M.; Crawford, J.M.; McGinn, T.; Davidson, K.W. Presenting Characteristics, Comorbidities, and Outcomes Among 5700 Patients Hospitalized with COVID-19 in the New York City Area. JAMA 2020, 323, 2052-2059. [CrossRef]

54. Goyal, P.; Choi, J.J.; Pinheiro, L.C.; Schenck, E.J.; Chen, R.; Jabri, A.; Satlin, M.J.; Campion, T.R., Jr.; Nahid, M.; Ringel, J.B.; et al. Clinical Characteristics of COVID-19 in New York City. N. Engl. J. Med. 2020, 382, 2372-2374. [CrossRef]

55. Guan, W.-J.; Ni, Z.-Y.; Hu, Y.; Liang, W.-H.; Ou, C.-Q.; He, J.-X.; Liu, L.; Shan, H.; Lei, C.-L.; Hui, D.S.C.; et al. Clinical Characteristics of Coronavirus Disease in China. N. Engl. J. Med. 2020, 382, 1708-1720. [CrossRef]

56. Huang, C.; Wang, Y.; Li, X.; Ren, L.; Zhao, J.; Hu, Y.; Zhang, L.; Fan, G.; Xu, J.; Gu, X.; et al. Clinical features of patients infected with 2019 novel coronavirus in Wuhan, China. Lancet 2020, 395, 497-506. [CrossRef]

57. Bhatraju, P.K.; Ghassemieh, B.J.; Nichols, M.; Kim, R.; Jerome, K.R.; Nalla, A.K.; Greninger, A.L.; Pipavath, S.; Wurfel, M.M.; Evans, L.; et al. COVID-19 in Critically Ill Patients in the Seattle Region-Case Series. N. Engl. J. Med. 2020, 382, $2012-2022$. [CrossRef]

58. Zhou, F.; Yu, T.; Du, R.; Fan, G.; Liu, Y.; Liu, Z.; Xiang, J.; Wang, Y.; Song, B.; Gu, X.; et al. Clinical course and risk factors for mortality of adult inpatients with COVID-19 in Wuhan, China: A retrospective cohort study. Lancet 2020, 395, $1054-1062$. [CrossRef]

59. Lu, C.; Liu, Y.; Chen, B.; Yang, H.; Hu, H.; Zhao, Y. Prognostic value of lymphocyte count in severe COVID-19 patients with corticosteroid treatment. Signal Transduct. Target. Ther. 2021, 6, 106. [CrossRef]

60. Van Paassen, J.; Vos, J.S.; Hoekstra, E.M.; Neumann, K.M.I.; Boot, P.C.; Arbous, S.M. Corticosteroid use in COVID-19 patients: A systematic review and meta-analysis on clinical outcomes. Crit. Care 2020, 24, 696. [CrossRef] 